\title{
Differential Antibacterial Activity of the Various Crude Leaf Extract of Eucalyptus Offensiveness Against Selected Pathogenic Bacterial Strains
}

\author{
Nwankwo, I.U. and Ukaegbu-Obi, K.M. \\ Department of Microbiology, College of Natural Science, Michael Okpara University of Agriculture Umudike, \\ PMB 7267 Umuahia, Abia State, Nigeria.
}

\begin{abstract}
The antibacterial activities of Eucalyptus officinalis extracts were studied. The antibacterial efficiency of the above mentioned plant was evaluated according to agar diffusion and broth dilution methods by using Escherichia coli, Staphylococcus aureus, Streptococcus pneumoniae, Proteus mirabilis and Pseuomonas aeruginosa clinical isolates and typed cultures. The most susceptible bacteria were E. coli followed by S. aureus while the most resistant bacteria were Pseud. aeruginosa followed by P. mirabilis and Strept. Pneumoniae. From the screening experiment, the hot water extract produced the highest inhibitory effect $17.22 \pm 0.14$ and $17.63 \pm 0.06$ against the clinical isolated and typed culture respectively, hence this plant can be further subjected to isolation of the therapeutic antimicrobials and pharmacological evaluation.
\end{abstract}

Keywords: antibacterial activity, bacterial strains, crude leaf extract, Eucalyptus officinalis, pathogenic,

\section{Introduction}

Medicinal plants are a source of great economic value all over the world (Bishmu et al, 2009). A large number of diverse types of plants grow in different parts of the country. Nigeria, being rich with all the three levels of biodiversity, namely species diversity, genetic diversity and habitat diversity possess thousands of species with known medicinal value and the use of different parts of these medicinal plants to cure specific ailments has been in vogue since ages.

The increase in antibiotic resistance bacteria is largely due to the widespread use of antibiotics in medicine, in animal care, and in agriculture. The problem is compounded by the lack of new antibiotics to attack bacteria in different ways to circumvent the resistant genes. Decreasing efficiency and resistance of pathogens to antimicrobial drugs made the search of a new antimicrobial agent an important strategy for the establishment of alternative therapies in difficult handling infections.

Plants are potent biochemists and has been components of phyto-medicine, a wondrous assortment of industrial chemicals have been obtained from them (Parekh et al, 2006).

However, there has been a rising interest in the research for natural products from plants for the discovery of new antimicrobial and antioxidant agents in recent times (Nascimento et al, 2000, Rios and Recio, 2005). Moreso, many of these plants have been known to synthesize active secondary metabolites such as phenolic compounds found in essential oils with established potent insecticidal and antimicrobial activities, which indeed has formed the basis for their applications in some pharmaceuticals, alternative medicine and natural therapies (Reynolds, 1996; R'ios and Recio, 2005).

Santo et al (1997) remarked that the World Health Organization (WHO) has indeed recognized medicinal plants as the best source for obtaining a variety of synthetic drugs. Some studies have identified and isolated the main active ingredients in the plants responsible for this antimicrobial activity Oloke et al 1988; Carson and Riley, 1995). However, the study on medicinal plants will allow for the demonstration of their physiological activity and also catalyze many pharmacological studies that will lead to the development of more potent drugs with no or minimal toxicity and high sensitivity especially towards the emerging microbial agents Fabricant and Fansworth, 2001). The main objective of the research is to screen and evaluate antibacterial activity of crude extracts and to find out minimum bactericidal concentration (MBC) against both gram positive as well as gram negative.

\section{Materials and Methods}

2.1 Aqueous and ethanolic extraction of the plant parts: Modified Okogun (2000) method of extraction was adopted in the process. Such that the diluents used were $95 \%$ ethanol and hot water. 50g of plant material were boiled with $200 \mathrm{ml}$ of water and allowed to cool for the aqueous extraction. For ethanol extraction, $15 \mathrm{~g}$ of powdered plant material was soaked in $150 \mathrm{ml}$ of ethanol for $24 \mathrm{~h}$. The filtrate was concentrated at $45^{\circ} \mathrm{C}$ under reduced pressure using a rotary vacuum evaporator. The extracts were kept at refrigeration temperature until required for use. 
2.2 Phytochemical screening: Phytochemical screening of the various plant extract was carried out using standard procedure as described by Adegoke and Adebayo (2009a and b) and Trease and Evans (1984) to determine the presence of alkaloids, saponins, tannins, anthraquones, glycoside, flavonoids and reducing sugar.

2.3 Collection of test organism: Test organisms (Staphylococcus aureus, Escherichia coli, Streptococcus pneumoniae, Proteus mirabilis and Pseudomonas aeruginosa) were obtained from Federal Medical Center Owerri Microbiology Laboratory, while the type cultures were obtained from Federal College of Veterinary and Medical Technology, Vom, Jos, Plateus State. The identity of the test organisms were reconfirmed and stored at $4^{\circ} \mathrm{C}$ in nutrient agar slant.

2.4 Sensitivity screening: Agar well diffusion method was used to determine the preliminary sensitivity screening of the crude plant extract. The standardized innoculum of the test organism using Mcfarland standard was seeded on sterile Muller Hinton agar plants. Duplicate wells of $5 \mathrm{~mm}$ were made using a corck borer. $100 \mathrm{ml}$ of $200 \mathrm{mg} / \mathrm{ml}$ concentration of the various plant extracts was introduced into the wells. The plates were allowed to stand for 1 hour for diffusion to take place and then incubated for $24 \mathrm{~h}$ at $37^{\circ} \mathrm{C}$. Using a ruler the zone of inhibition was measured to the nearest millimeters.

2.5 Determination of the minimum inhibitory concentration (MIC): Using the macro-broth method as described by NCCLS (1998), a two-fold serial dilution of the reconstituted extract was prepared using Mueller Hinton Broth. Each dilution was inoculated with $100 \mu$ l of the standardized suspension of the test organism, the cultures were incubated at $37^{\circ} \mathrm{Cfor} 24 \mathrm{~h}$. The MIC was determined as the highest dilution that showed visible growth.

2.6 Determination of minimum bactericidal concentration (MBC): $0.1 \mathrm{ml}$ of all MIC tubes showing no growth was inoculated into sterile Mueller Hinton agar plate incubated at $37^{\circ} \mathrm{C}$ for $24 \mathrm{~h}$ and observed for growth. MBC growth was determined at least concentration showing no growth.

2.7 Statistical analysis: The inhibition zones were statistically analyzed by conducting a paired sample Ttest using SPSS (2006) computer application programmes. Means \pm standard deviation were considered significant at $\mathrm{P}=0.05$.

\section{Results}

Amongst the phytochemicals tested, only anthraquones and glycosides were not detected in both the ethanolic and hot water extract of E. officinals. This is shown in Table 1. In Table 2, it was observed that hot water extract of $E$. officinalis had the highest inhibitory effect against both the clinical isolates and typed culture of Escherichia coli (17.22 \pm 0.14 and $17.63 \pm 0.06$ respectively). This was followed by the ethanol extract against Staphylococcus aureus. Proteus mirabilis was slightly inhibited by both the ethanol and hot water extracts.

From the results of the MIC and MBC of the extracts, it was observed that the ethanol extract exhibited a bactericidal effect against both the clinical isolate and typed culture of S. aureus and Escherichia coli. The hot water extract also showed a bactericidal effect against Escherichia coli. Both extracts, showed a bacteriostatic effect against the clinical isolate and typed culture of P.mirabilis. This is as shown in Table 3.

\section{Discussion}

Plant extracts are valuable resources for primary health care and complementary health care system. Undoubtedly plant extracts containing substances of medicinal value that are yet to be discovered, though large number of plant extracts are constantly being screened for their antimicrobial effect, these plant extracts may prove to be rich source of compounds with possible antimicrobial activities.

The phytochemical screening of the ethanolic and aqueous extracts of Eucalyptus officinalis did show the presence of mainly phenolic compounds (alkaloids, saponins, tannins, flavonoid) which have been found to possess antimicrobial properties (Alma et al, 2003). Several studies have described the antioxidant properties of medicinal plant extracts which are rich in phenolic compounds such as flavonoids, Quinones etc. Phenolic compounds have been shown to be toxic to microorganisms. The mechanism thought to be responsible for phenolic toxicity to microorganisms includes enzyme inhibition by the oxidized compound possible through reaction with sulflydral groups or through more nonspecific interactions with the protein (Manson, 1987).

The results of antimicrobial activity and MC arrays showed promising evidence for the antimicrobial activity of E. officinalis fresh leaf extracts against some human pathogens. The low zone of inhibition obtained with some extracts particularly the ethanol extract may be due to errors or contamination.

Comparing the sensitivity of the clinical isolates to the typed cultures as shown in Table 2, it can be deduced that the typed cultures exhibited relatively higher degree of sensitivity to the crude extracts. This may be because the clinical isolates may have developed some degree of resistance due to continuous exposure to antibiotic agents. This is in line with the observation of Hugo et al (1991), that the continuous presence of antibiotic can cause microorganisms to alter their metabolic pathways or produce an exflux pump system that can pump antibiotic out of the microbial cells. 
Pseudomonas aeroginosa resistance to antimicrobials is through alteration of cell membrane permeability possibly by modification of protein the outer membrane, so that it is difficult for an antimicrobial to enter the bacterial cell and also its tendency to colonize surfaces in a biofilm form thereby making the cell impervious to therapeutic concentration of antibiotics. The observed insensitivity of Pseudomonas aaeruginosa to most of the plant extracts agreed with similar work done by Bishu et al (2009) and Shittu et al (2006) using alcoholic and aqueous plant extracts.

Considerable antibacterial activities of the plant sample were noted in the various extracts as compared to the standard antibiotic penicillin. The results revealed the presence of medicinally important constitutes in the E. officinales leaf extracts.

\section{Conclusion}

Many evidences gathered in earlier studies which confirmed the identified phytochemicals to be bioactive. Therefore these E. officinales leaf extracts could be seen as a good source for useful drugs and have great potentials as antimicrobial agents against selected pathogens and they can be used as alternative medicine in the treatment of infections caused by these strains of bacteria.

\section{References}

[1] Bishmu, J.S., Unil, L. and Anuja, S. Antibacterial property of different medicinal plants: Ocinum santum, Cinnamonum zeylamicum, Xanthoxylum armatum and Origannan majorana. Kathmandu Uni.. J. Sci. Engr. Tech. 5(1) (2009): 143-150.

[2] Parek, J., Karathia, N. and Chande, S. Evaluation of antibacterial activity and phytochemical analysis of bauhinia variegate L. bark. African. J. of Biomedical Research. 9: (2006): 53-56.

[3] Nascimento, G.G., Locateli, J. Frectas, P.C. and Gruliana L.S. Antibacterial activity of plant extracts and phytochemicals on antibiotic resistant bacteria: Brazilian Journal of Microbiology. 31: (2000): 247-256.

[4] R'ios, J.L. and Recio, M. Medicinal plants and antimicrobial activity. Journal of Ethnopharmacology. 100: (2005): 80-84.

[5] Reynolds, J.E.F. Matindale-the extra pharmamcopia. $31^{\text {st }}$ edition. (Royal pharmaceutical society of Great Britain, London 1996)

[6] Santo, Y.. Odetani, H., Singyouchi, K., Ohtsubo, T. Kihara, M. Shibats, H. and Higuti, T. Extraction and purification of effective antimicrobial constituents of Terminalis chebulla RETS against methicillin-resistance Staphylococcus aureus. Boil. Pharm. Bull. 20: (1997):401-404.

[7] Oloke, J.K., Kolawo, B.O. and Erhun, W.O. Antibacterial and antifungal activities of certain components of Afranomum mallegueta Fitoterapia 59: (1988) 384-388.

[8] Carson, C.F. and Riley, T.V. Antimicrobial activity of the major components of the essential oil of Melaleuca alternifolia. J. Appl. Bacteriol. 78: (1995) 264-269.

[9] Fabricant, D.S. and Farnsworth, N.R. The value of plant used in traditional medicine for drug discovery. Environmental Health prspective supplements. 109: (2001) 69-75

[10] Okogun, J.I.. Methods of medicinal plants extracts preparation. (National institute of pharmaceutical research and development (NIPRD) Idu-Abuja, Nigeria 2000)

[11] Adegoke, A.A. and Adebayo-tayo, B.C. Antibacterial activity and phytochemical analysis of leaf extracts of Lasienthera africanum. Afr. Journ. of Biotech. 8 (1): (2009a):077-080.

[12] Adegoke, A.A. and Adebayi-tayo, B.C. Phytochemical composition and antimicrobial effects of Corchores olitorius leaf extracts on four bacterial isolates. J. of Med. Plants Res. 3(3): (2009b) 155-159.

[13] Trease, G.E. and Evans, W.C. Textbook on pharmacology $12^{\text {th }}$ edition (Balliere Tindel and company publishers, London. 1984) 343-383.

[14] National committee for clinical laboratory standard (NCLS) Methods for dilution in antimicrobial susceptibility. Test villanoova. Ninth Information Suppl. 25: (1998)23-29.

[15] SPSS Statistical Package for Social Sciences. (SPSS 15.0 for Window Evaluation version. 2006).

[16] Alma, M.H., Mavi, A. Yildirim, A., Digrak, M. and Hirata, T. Screening chemical composition and in-vitro antioxidants and antimicrobial activities of the essential oils from the Origanum syriacum L, growing in Turkey. Biol. Pharmaceu. Bull. 26: (2003) 1725-1729.

[17] Manson, T.L. Inactivation of red beet beta-glucan synthase by native and oxidized phenolic compounds. Phytochem. 26: (1987). 2197-2202.

[18] Hugo, W.C., Russel, A.D. and Brown, S.S.. Pharmaceutical microbiology (Blackwell Scientific publication, Oxford 1991 ) $265-274$.

[19] Shittu, L.A.J., Bankole, M.A., Ahmed, T., Aile, K. Akinsanya, M.A., Bankole, M.M., Shittu, R.K. and Ashiru, O.A. Differential antimicrobial activity of the various crude leaves extracts of Sesame radiatum against some common pathogenic microorganisms. Sci. Res. Essay. 1(3) (2006) 108-111.

TABLE 1: Phytochemical screening result of Eucalyptus offinalis extracts

\begin{tabular}{|l|l|l|}
\hline Phyto constituents & Ethanol extract & Hot water extract \\
\hline Alkaloid & + & + \\
\hline Saponins & + & + \\
\hline Flavonoids & - & + \\
\hline Tannins & + & - \\
\hline Anthraquone & - & - \\
\hline Glycosides & - & - \\
\hline Reducing sugar & + & + \\
\hline
\end{tabular}


Differential Antibacterial Activity of the Various Crude Leaf Extract of Eucalyptus Offensiveness

TABLE 2: Mean zone of inhibition of Eucalyptus officinalis extracts

\begin{tabular}{|l|l|l|l|}
\hline Organisms & Ethanol extract & Hot water extract & Pecicillin \\
\hline Escherichia coli & & & \\
\hline Clinical isolate & $7.05 \pm 0.01$ & $17.22 \pm 0.14$ & $24.78 \pm 0.08$ \\
\hline Typed culture (ATCC 1775) & $9.06 \pm 0.01$ & $17.63 \pm 0.06$ & \\
\hline Staphylococcus aureus & & & \\
\hline Clinical isolate & $8.81 \pm 0.08$ & $0.00 \pm 0.00$ & $13.17 \pm 0.09$ \\
\hline Typed culture (ATCC 12600) & $11.09 \pm 0.01$ & $1.48 \pm 0.74$ & \\
\hline Streptococcus pneumoniae & & & $13.73 \pm 0.06$ \\
\hline Clinical isolate & $0.00 \pm 0.00$ & $0.00 \pm 0.00$ & \\
\hline Typed culture (ATCC 6674) & $2.15 \pm 0.21$ & $0.70 \pm 0.07$ & \\
\hline Proteus mirabilis & & & $9.07 \pm 0.02$ \\
\hline Chemical isolate & $1.12 \pm 0.06$ & $1.46 \pm 0.07$ & \\
\hline Typed culture (NCTB 67) & $1.65 \pm 0.04$ & $2.17 \pm 0.03$ & $4.49 \pm 0.01$ \\
\hline Pseudomonas aeruginosa & & & \\
\hline Clinical isolate & $0.00 \pm 0.00$ & $0.00 \pm 0.00$ & \\
\hline Typed culture (ATCC 10145) & $0.19 \pm 0.01$ & $0.00 \pm 0.00$ & \\
\hline
\end{tabular}

TABLE 3: Minimum inhibitory concentration (MI) and minimum bactericidal concentration (MB) of the crude extracts of Eucalyptus officinals $(\mathrm{mg} / \mathrm{ml})$

\begin{tabular}{|c|c|c|c|c|c|c|}
\hline Organisms & Extra & & & & & \\
\hline & Ethan & tract & & Hot w & r extra & \\
\hline & MIC & $\mathrm{MBC}$ & MIC/MBC & MIC & MBC & $\mathrm{MIC} / \mathrm{MBC}$ \\
\hline Escherichia coli & & & & & & \\
\hline Clinical isolates & 25 & 50 & 0.50 & 100 & 100 & 1.00 \\
\hline Typed culture (ATCC 1775) & 25 & 50 & 0.50 & 50 & 50 & 1.00 \\
\hline Staphylococcus aureus & & & & & & \\
\hline Clinical isolates & 100 & 100 & 1.00 & $>200$ & $>200$ & $<0.50$ \\
\hline Typed culture (ATCC 12600) & 100 & 100 & 1.00 & $>200$ & $>200$ & $<0.50$ \\
\hline Streptococcus pneumoniae & & & & & & \\
\hline Clinical isolates & $>200$ & $>200$ & $<0.50$ & $>200$ & $>200$ & $<0.50$ \\
\hline Typed culture (ATCC 6674) & 100 & $>200$ & $<0.50$ & 200 & $>200$ & $<0.50$ \\
\hline Proteus mirabilis & & & & & & \\
\hline Clinical isolates & 100 & $>200$ & $<0.05$ & 100 & $>200$ & $<0.50$ \\
\hline Typed culture (NCTB 67) & 100 & $>200$ & $<0.05$ & 100 & $>200$ & $<0.50$ \\
\hline Psedomonas aeruginosa & & & & $>200$ & $>200$ & $<0.50$ \\
\hline Clinical isolates & $>200$ & $>200$ & $<0.50$ & $>200$ & $>200$ & $<0.50$ \\
\hline Typed culture (ATCC 10145) & $>200$ & $>200$ & $<0.50$ & & & \\
\hline
\end{tabular}

